\title{
Kyphosis index obtained in X-ray and with flexicurve assessment in children and young people
}

Rafael Paiva Ribeiro 1

Bárbara Vendramini Marchetti 2

Eduardo Bojunga de Oliveira 3

Claúdia Tarragô Candotti 4

\footnotetext{
1-4 Universidade Federal do Rio Grande do Sul. Av. Paulo Gama, 110. Farroupilha. Porto Alegre, RS, Brasil. CEP: 90.040-060. E-mail: rpaivaribeiro2@gmail.com
}

\begin{abstract}
Objectives: to assess the concurrent validity of kyphosis index measured in the flexicurve from the correlation of $X$-rays exams, identifying its accuracy and to assess the thoracic spine in children and young people.

Methods: 31 young people at an average age of 11.1 3.4 years were evaluated by digital x-rays: (1) Cobb angle (ÂngCobb), (2) Kyphosis index (KIX), and (3) KIX angle (ÂngKIX). These were measured from the flexicurve design on the millimetric graph paper: (1) Kyphosis index (KIFint), obtained from C7 the intersection of kyphosis-lordosis, (2) Kyphosis index (KIFT12), obtained between C7 and T12, and (3) Kyphosis angle (ÂngKIFint and AngKIFT12). Statistical analysis: Correlation to Pearson Moment-Product and t test $(\alpha<0.05)$.

Results: the angular values (ÂngKIFint, AngKIFT12, AngKIX) were underestimated in relation to Cobb angle $(p<0.05)$, correlating only to KIX angle and Cobb angle [ $r=0.698$, $p<0.001]$. The linear values (KIFint, KIFT12, KIX) were similar $(p>0.05)$ among themselves, correlating only to KIX and Cobb angle [r=0.698, $p<0.001]$ and KIX angle and KIX [r=1; $p<0.001]$.

Conclusions: the KIX and KIX angle presented as an accurate method and valid to be used in the thoracic kyphosis assessment, although KIFint, KIFT12, KIFint angle and KIFT12 angle showed no correlation to the gold standard and not being indicated to assess the thoracic kyphosis in children and young people.
\end{abstract}

Key words Kyphosis, Posture, Validity of tests, X-rays 


\section{Introduction}

The Cobb angle (ÂngCobb) is considered as the standard method to assess the vertebral column through $\mathrm{x}$-rays examinations. ${ }^{1-5}$ Nevertheless, these exams are contraindicated in some population, as for people with cancer, pregnant women 6 and children in the development phase, ${ }^{7}$ in addition to being a high cost procedure for underdeveloped and developing countries. ${ }^{8}$

Thus, techniques for assessing noninvasive spine have been proposed as they are considered to be of low complexity and low cost. In this context, instruments such as the photogrammetry associated to softwares ${ }^{9}$ the arcometer $^{10}$ and the flexicurve 8 have driven to new researches on postural assessment in order to promote the use of these low complexity systems. The flexicurve already well documented in the literature, consists of a straight ruler, made of plastic-coated metal, which is molded on the back of the individual in order to illustrate the outline of the vertebral column, allowing a quick and cheap assessment of the curvatures of the vertebral column in the clinical environment, and also in study fields or with a big population. 8 Therefore, the flexicurve can constitute of a useful tool, especially in monitoring the children and young people's postural treatment, which requires frequent assessments because they are in the development stage 7 and they should not be exposed repeatedly to the deleterious effects of Xray examinations. 6,7

Considering the contour assessment of the vertebral column, the flexicurve can provide angular and linear measurements representing the curvatures. As for the measurement of the angle, the flexicurve has already been validated in relation to the gold standard for both the thoracic and lumbar. ${ }^{8}$ Nevertheless, up to where it is known, the linear measurements have been suggested for the population of adults and there is still a investigation gap with the population of children. The linear measurements involve width and length of the curvature and the reasons for such measurements in the thoracic spine, generates an index, called kyphosis index. ${ }^{11}$

The importance in the use of kyphosis index (KI) is in its simplicity and practicality, since the professional can readily calculate from the molding of the individual's column by getting an immediate assessment result. However, for KI to be indeed an assessment tool, it needs to know its psychometric properties, such as its accuracy and precision. Therefore, the objective of this study is to evaluate the concurrent validity of the kyphosis index (KI) measured with the flexicurve according to the X-ray exams, identifying its accuracy to assess the children and young people's thoracic spine.

\section{Methods}

The sample was defined by a sample calculation using Gpower 3.1.7software, providing a one-tail test; a null hypothesis corresponding to the correlation of value $r$ of 0.4 (any correlation value below 0.4 is clinically unacceptable); an expectation of moderate correlation $(\mathrm{r}=0.6)$; a size of great effect ( $\mathrm{f}=0.5$ ); an error $\alpha=0.05$ and a power of $80 \%$ resulting in a minimum sample size of 22 participants. Expecting losses, 31 young people who had performed X-rays exams in a hospital in Porto Alegre were invited to participate of this study. The inclusion criteria were to be a child or adolescent, both genders and aged between 6 and 16 years. The young people who had previous surgery of the column or congenital deformities were excluded. This study was approved by the Ethics Committee in Research at the University where the study was conducted (Number: 675,873), and those responsible for the young people signed a term of consent.

The young people were assessed in a single day, although the X-rays and the flexicurve assessment were performed sequentially. All the assessment with the flexicurve was conducted by researcher A and the $\mathrm{x}$-rays were performed by a responsible technician from the hospital. In the panoramic x-ray examination of the spine in the sagittal plane was always followed by the same protocol: (1) positioning the young person in orthostasis position in a normal posture, (2) upper limbs in shoulder flexion $\left(90^{\circ}\right)$, (3) requesting the young person to remain steady.

From the x-rays exams, researcher $\mathrm{B}$ had done the calculations of Cobb angles (ÂngCobb) by using the four lines methodology 12 by mathematics routine of Matlab ${ }^{\circledR} 7.9$ software specially developed for this study. To calculate the kyphosis thoracic angle, the superior vertebral plateau of $\mathrm{T} 1$ and the inferior vertebral plateau of T12 were marked (Figure 1A). Still, from the x-rays, also using a routine of Matlab software ${ }^{\circledR} 7.9$, the same researcher calculated the kyphosis index of the X-rays exams (KIX) by using the spinous process of $\mathrm{C} 7$, the spinous process of T12 and the spinous process of the vertebra apex of the curvature (Figure 1B).

The flexicurve assessment was performed shortly after the $\mathrm{x}$-rays. The procedures were performed according to Oliveira et al., ${ }^{8}$ while the children had no clothes on their backs, the researcher began to touch and mark the spinous processes (PE) 
of the vertebrae $\mathrm{C} 7, \mathrm{~T} 1, \mathrm{~T} 12, \mathrm{~L} 1, \mathrm{~L} 5$ and $\mathrm{S} 1$ with stickers and requested that the child would stay in the same position as performed in the X-ray room, with their feet in the width of the hips, shoulders and elbows flexed at $90^{\circ}$ and sustained on the wall so that the flexicurve could be molded on his/her back on the vertebral column. Afterwards, the flexicurve was removed from his/her back and placed on a millimetric graph paper where the curvatures were drawn and the spinous process was marked on paper. Using a routine in Matlab ${ }^{\circledR} 7.9$ software, researcher $\mathrm{C}$ calculated the kyphosis index (KI) from the contour of the column drawn on a millimetric graph paper, using the spinous process of $\mathrm{C} 7$, the spinous process of $\mathrm{T} 12$ and the spinous process of the apex vertebral curvature. Both indexes were calculated: (1) the intersection of the kyphosis index (KIFint), whereas the spinous process of $\mathrm{C} 7$, the intersection between the thoracic and lumbar curvatures and the apex of the thoracic curvature (Figure 1C); (2) Kyphosis index T12 (KIFT12) whereas the spinous process of $\mathrm{C} 7, \mathrm{~T} 12$ was marked and the apex thoracic curvature (Figure 1d). The calculation on the three kyphosis indexes (KIX, KIFint, KIFT12) was performed according to Hinmann, 11 by using Equation 1.

$$
K I=\frac{\text { width }}{\text { length }} \times 100 \quad \text { Equation } 1
$$

In possession of the three kyphosis indexes (KIX, KIFint and KIFT12), they were inserted in the Equation 2, proposed by Greendale et al. 13 Thus, it was possible to obtain an angular value for kyphosis (AngKI), from KI, in other words, the values of KIX angle (predicted angle from KIX) KIFint angle (predicted angle from KIFint) and KIFT12 angle (predicted angle from KIFT12).

$$
\operatorname{Ang} K I=(314,61 \times K I)+5,11 \quad \text { Equation } 2
$$

The data were analyzed by using the Statistical Package for Social Sciences (SPSS), version 18.00. Initially, the normality of the data was verified through the Shapiro-Wilk test. For all linear and angular variables were used the following tests: Pearson Product-Moment correlation and t-Test independent. Still, the RMS error (Root Mean Square) was calculated to estimate the accuracy of the measurements, because it corresponds to a correlation measurement between the estimate and the real parameter value, in other words, the RMS error reflects on how much the estimated value is close to the real value. Pearson coefficient ( $r$ ) was classified as null (0), weak (between 0 and 0.3 ), moderate (between 0.30 and 0.6), strong (between 0.6 and 0.9 ), very strong (between 0.9 and 1) and perfect (1), according to Callegari-Jacques. 14 The significance level was 0.05 .

Cobb angle - CA (A); Kyphosis index in the X-rays exams - KIX (B); Kyphosis index of the flexicurve - KIFint (C) and Kyphosis index in the flexicurve - KIFT12 (D).

A

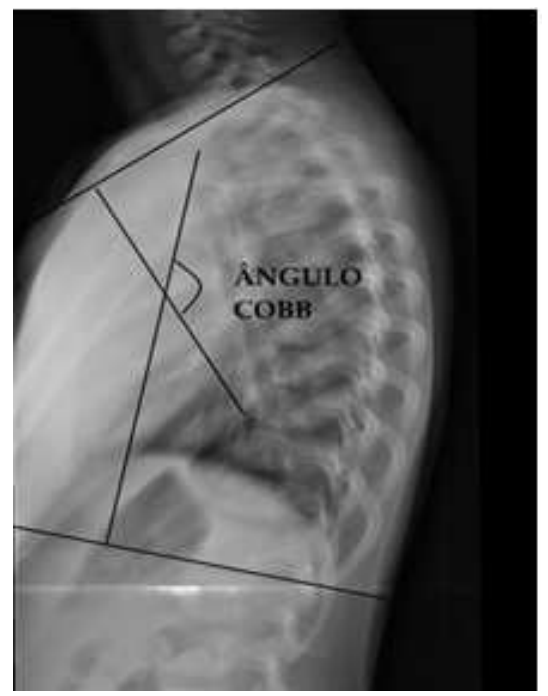

B

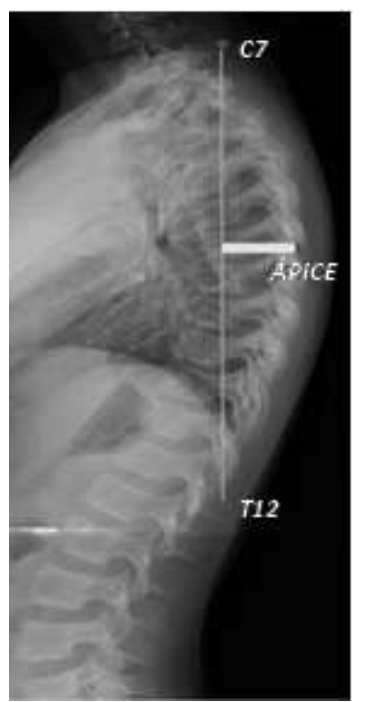

C

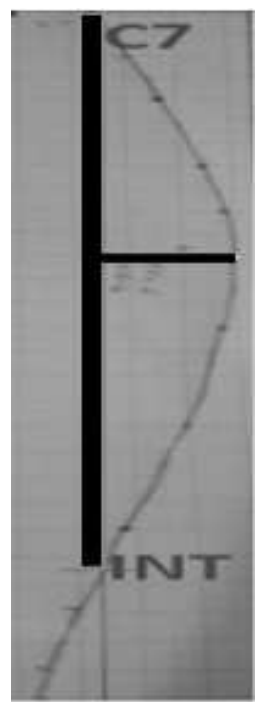

D

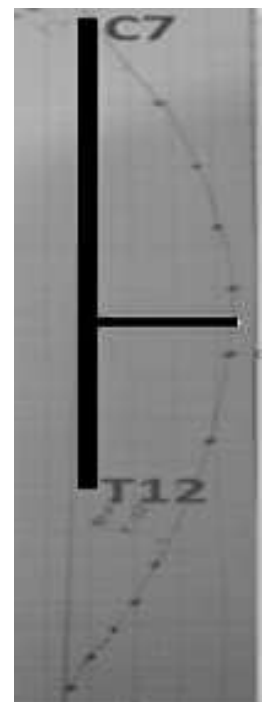




\section{Results}

The sample was composed of 31 children and young people with an average age of $11.1+3.4$ years, in which $58 \%(n=18)$ were females and $42 \%(n=13)$ males. The values of KI angle (AngKI) (AngKIX, AngKIFint, AngKIFT12) were underestimated in relation to the values of Cobb angle (AngCobb), with significant differences between these measurements (Table 1). Since the values of KIFint and KIFT12 were similar in relation to KIX (Table 1). The RMS error calculated for KIX, KIFint and KIFT2 angles (ÂngKIX, ÂngKIFint, ÂngKIFT2) in relation to Cobb angle was $10.7^{\circ}, 12.7^{\circ}$ and $15.5^{\circ}$, respectively.

In the correlation analysis tests, we found a strong correlation (Figure 2A) between KIX and Cobb angle (ÂngCobb) $(\mathrm{r}=0.698 ; p<0.001)$ and between KIX (ÂngKIX) and Cobb angles (ÂngCobb) $(\mathrm{r}=0.698 ; p<0.001)$ and a perfect correlation (Figure 3A) between KIX angle (ÂngKIX) and KIX ( $\mathrm{r}=1.00 ; p<0.001)$. All the flexicurve variables (KIFint, KIFT12, ÂngKIFint, ÂngKIFT12) showed no significant correlation with Cobb angle (ÂngCobb) (Figure 2B and 2C) and with KIX (Figure 3B and 3C).

Table 1

Mean and standard deviation (SD) of the kyphosis indexes (KIFint, KIFT12, KIX) and the angles of the thoracic curvature (AngCobb, AngKIFint, AngKIFT12, AngKIX).

\begin{tabular}{lccc}
\hline & $\bar{X} \pm$ SD & t & p \\
& & & \\
& & - & $<0,001$ a \\
ÂngCobb $\left(^{\circ}\right)$ & $44,3 \pm 9,2$ & 6,7 & $0,001 \mathrm{~b}$ \\
ÂngKIX $\left(^{\circ}\right)$ & $36,0 \pm 8,5$ & 3,6 & $0,014 \mathrm{c}$ \\
ÂngKIFint $\left(^{\circ}\right)$ & $37,3 \pm 7,0$ & 2,6 & - \\
ÂngKIFT12 $\left(^{\circ}\right)$ & $37,6 \pm 12,0$ & - & $0,442 \mathrm{~d}$ \\
KIX (dimensionless) & $9,8 \pm 2,7$ & $-0,8$ & $0,513 \mathrm{e}$ \\
KIFint(dimensionless) & $10,2 \pm 2,2$ & $-0,7$ & \\
KIFT12 (dimensionless) & $10,3 \pm 3,8$ & & \\
\end{tabular}

ÂngCobb: Cobb angle; KIFint: kyphosis index marked between C7 and the intersection in flexicurve; KIFT12: kyphosis index marked between C7 and T12 in flexicurve; KIX: kyphosis index marked between C7 and T12 in the X-rays; AngKIFint: kyphosis angle marked between $\mathrm{C7}$ and the intersection in flexicurve; AngKIFT12: kyphosis angle marked between $\mathrm{C7}$ and T12 in flexicurve; AngKIX: kyphosis angle marked between C7 and T12 in the x-rays.

Comparisons, " $t$ " test: a significant difference between AngCobb and AngKIX. b Significant difference between AngCobb and AngKIFint. c Significant difference between AngCobb and AngKIFT12. d Without significant difference between KIX and KIFint. e there was no significant difference between KIX and KIFT12. 
Correlations between Cobb Angle and: (A) the angles obtained with the Kyphosis indices (ÂngKIX, ÂngKIFint, ÂngKIFT12); (B) the Kyphosis indexes (KIX, KIFint); and (C) and the Kyphosis index of (KIFT12).

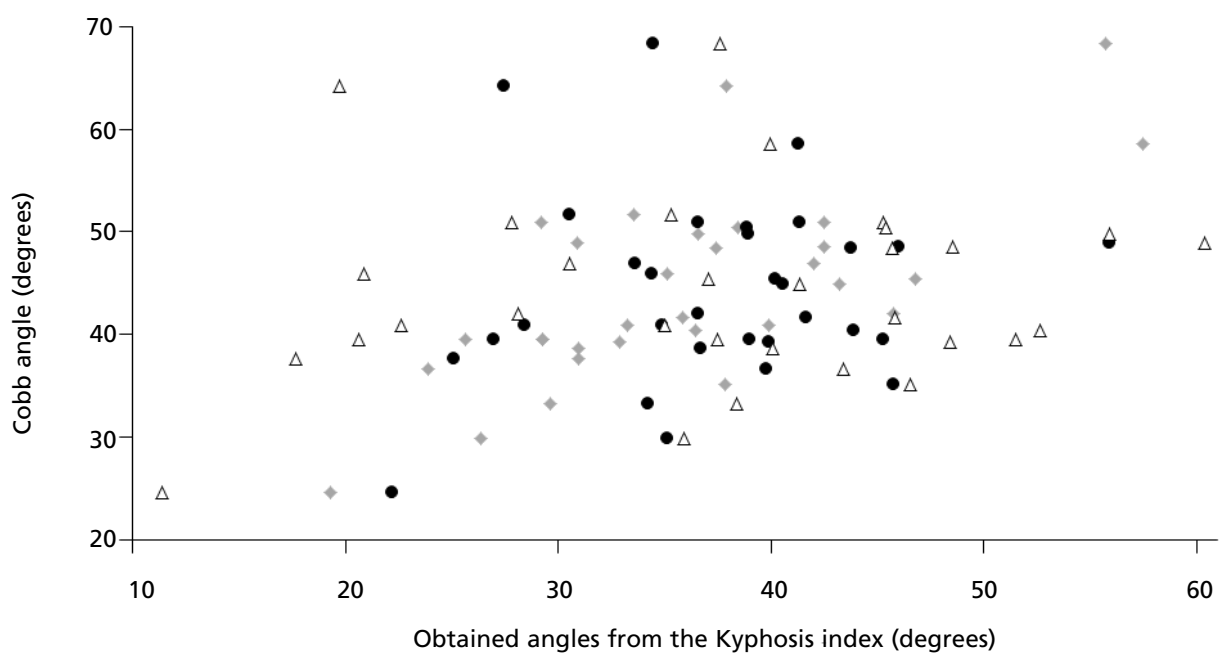

$$
\begin{array}{llll}
\text { cobb } \times \text { ÂngKIX } & \text { cobb } \times \text { ÂngKIFint } & \text { cobb } \times \text { ÂngKIFT12 } \\
(\mathrm{r}=0,698 ; p<0,001) & (\mathrm{r}=0,137 ; p=0,461) & (\mathrm{r}=0,127 ; p=0,495)
\end{array}
$$

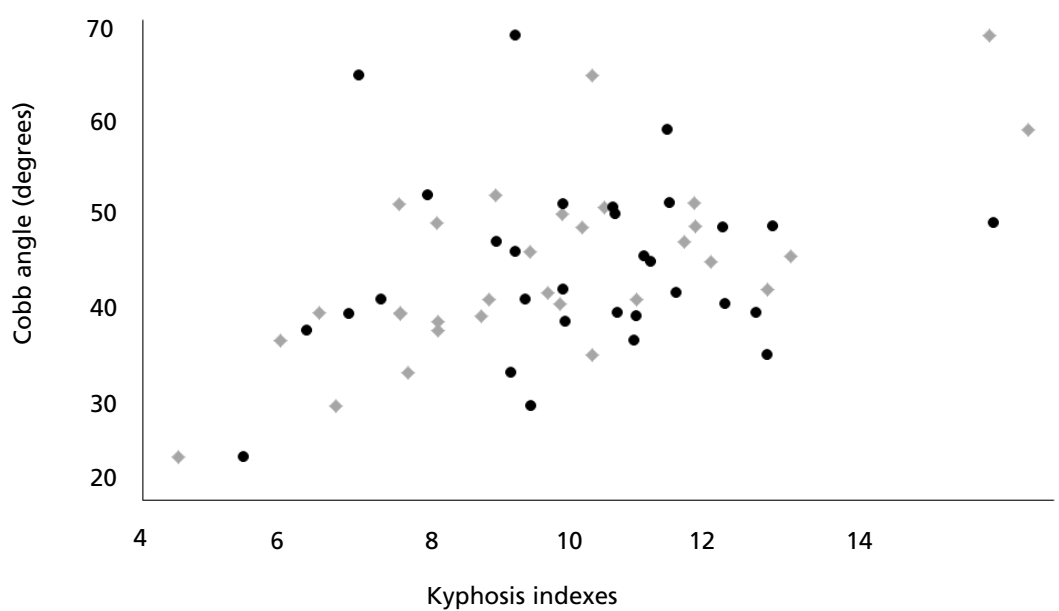

(B)

$$
\begin{array}{l|l}
\text { cobb } \times \mathrm{KIX} & \bullet \quad \text { cobb } \times \text { KIFint } \\
(\mathrm{r}=0,698 ; p<0,001) & (\mathrm{r}=0,137 ; p=0,461)
\end{array}
$$


Correlations between Cobb Angle and: (A) the angles obtained with the Kyphosis indices (ÂngKIX, ÂngKIFint, ÂngKIFT12); (B) the Kyphosis indexes (KIX, KIFint); and (C) and the Kyphosis index of (KIFT12).

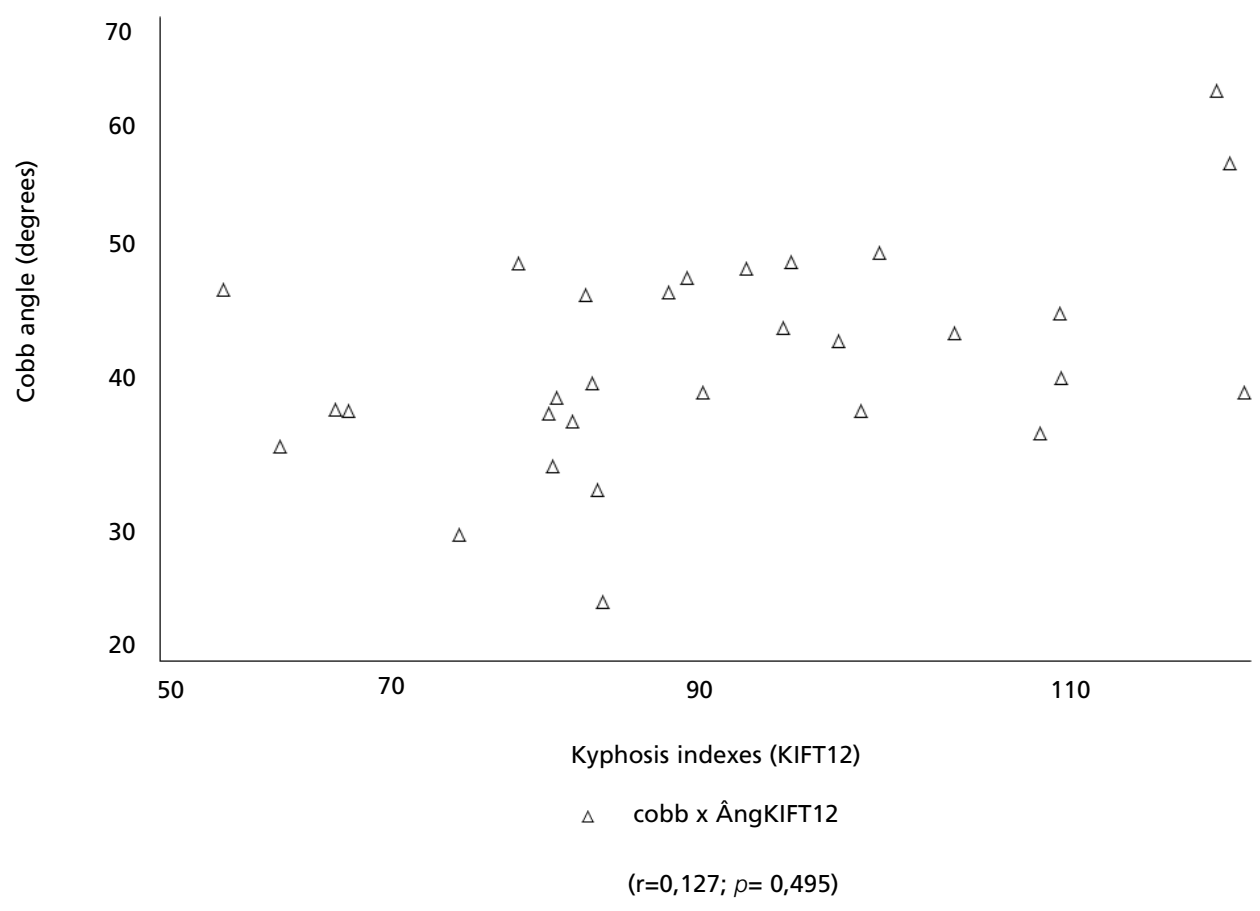

(C)

Figure 3

Correlations between the Kyphosis index of the X-ray (KIX) and: (A) the angles obtained from the Kyphosis indices (ÂngKIX, ÂngKIFint, ÂngKIFT12); (B) Kyphosis index of the Flexicurve (KIFint); and (C) and Kyphosis index of the Flexicurve (KIFT12).

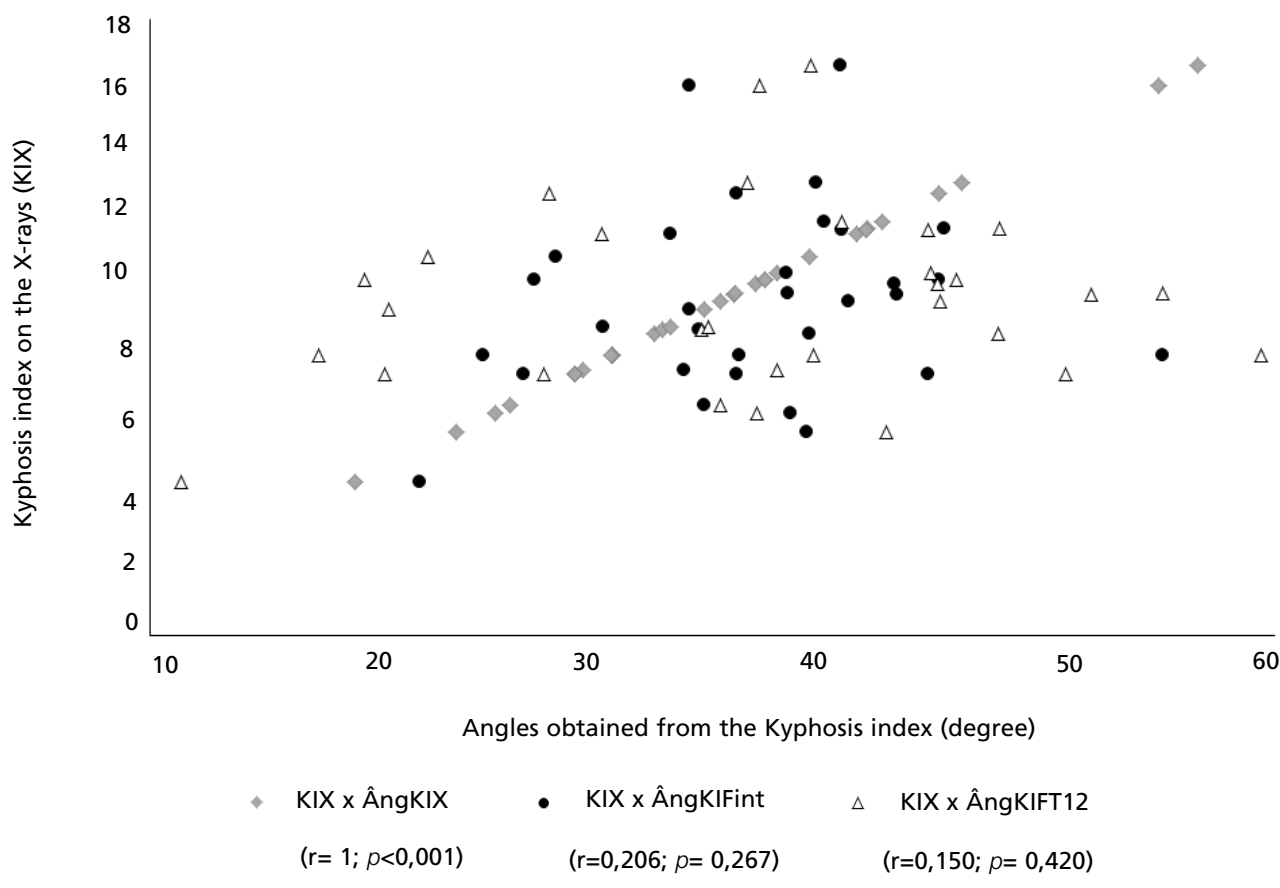


Correlations between the Kyphosis index of the X-ray (KIX) and: (A) the angles obtained from the Kyphosis indices (ÂngKIX, ÂngKIFint, ÂngKIFT12); (B) Kyphosis index of the Flexicurve (KIFint); and (C) and Kyphosis index of the Flexicurve (KIFT12).
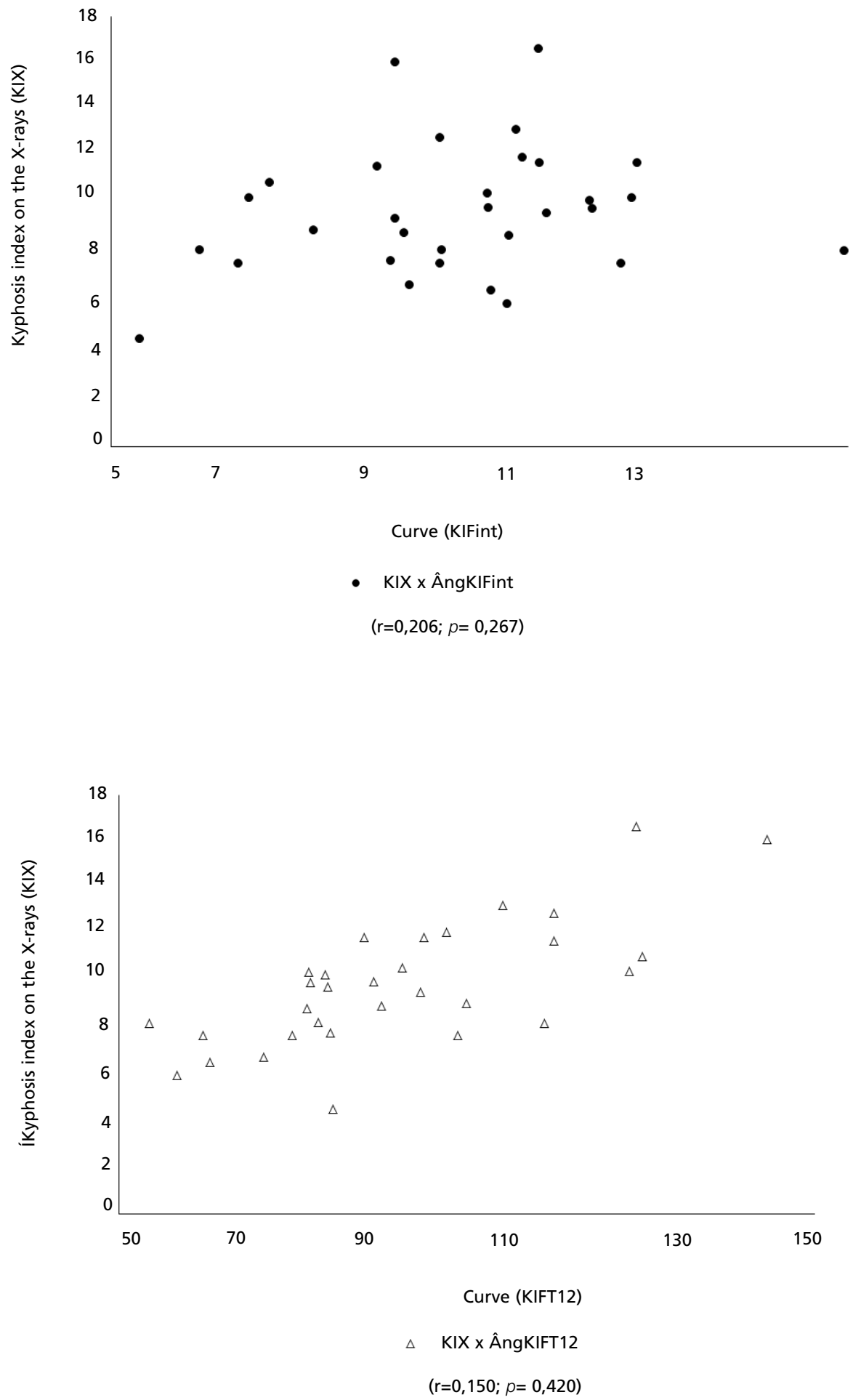


\section{Discussion}

Despite the broad spectrum of benefits that the flexicurve instrument provides for professionals in the health area, the findings of this study suggest that the linear and angular values provided by the flexicurve are not indicated for the evaluation of the thoracic kyphosis in children and young people, because they do not correlate with the gold standard and do not provide a accurate measurement of thoracic kyphosis. However, the kyphosis index results obtained in the X-ray examination (KIX) and the angle calculated from the KIX (ÂngKIX) constitutes in a possibility of accurately measuring children and young people's $\mathrm{x}$-ray assessment of the thoracic kyphosis.

The significant differences found between the angular values of the gold standard (ÂngCobb) with the flexicurve values (ÂngKIFint, ÂngKIFT12) are not present between the linear values of the gold standard (KIX) with the flexicurve values (KIFint, KIFT12) (Table 1). Perhaps, the form of calculating $\mathrm{KI}$, which is identical in both instruments (x-rays and flexicurve), which considers the same anatomical structures, may be responsible for similar results.

As for the analysis correlation, both the linear values (KIFint, KIFT12) and the angular values obtained by the flexicurve (ÂngKIFint, ÂngKIFT12) were not correlated to the gold standard (KIX, AngCobb). Harrison et al. 15 and McFarland et al. 16 highlight that some inherent problems to the method may be responsible for any errors in the calculations generated from the flexicurve. For example, the authors cite the smallest modifications of the flexicurve form, which are related to the inherent flexibility of the instrument and the fact of the severity, or a slight pressure, in the metal band contained inside might deform the instrument, before the precise line of trace is needed to be done on paper.

Thus, the imputed deformation to a flexible ruler, resulting from handling during the drawings of the backs until the transcription on the millimetric graph paper, could alternate the format of the curvature and with this the transcription would not in fact be realistic. In fact, the restless profile of children during the collecting procedure, since the moment of touching and marking specific points until the moment to mold the flexicurve, all of this may have contributed to errors in the measurement.

Still, according to Caine et al., ${ }^{17}$ the maximum length of the thoracic spine can be located in different locations of the arc, therefore, certain categories of kyphotic curves are not well represented by the kyphosis index (KI). Thus, a limitation of the present lays in the lack of tests with other limited vertebral. It is also important to emphasize that the children and young people has a body surface, as well as the size of the column (length and width), smaller in comparison to adults, increasing the chances of errors in the flexicurve instrument. A fact described in Harrison et al.,15 in which was found a weak assessment correlation of the flexicurve instrument with the X-ray examinations to evaluate the cervical region of the adults, finding it difficult in evaluating small regions.

As expected, analyzing the RMS errors, we found that the highest values were precisely for KIFT12 angle (ÂngKIFT12) and KIFint angle (ÂngKIFint), in which were not correlated with the gold standard, while the RMS error for KIX angle (ÂngKIX) presented a strong correlation with the Cobb angle, was considerably lower, at around the $10^{\circ}$. We did not find in any literatures in other paper to compare the relation of RMS errors of these variables, however, traditionally the values have been considered as clinically acceptable, in terms of reproducibility analysis in the Cobb angle, values between the $5^{\circ}$ and $10^{\circ}, 18$ in other words, the error between the gold standard measurements and the KIX angle (ÂngKIX) of $10,7^{\circ}$ could be considered acceptable.

Greendale et al.13 found a strong correlation between the kyphosis index and the Cobb angle, however the sample consisted of individuals aged 60 years or older, an age characteristic to present an increase of the curvature of the chest. Other studies $11,19,20$ that used the kyphosis index in the evaluation of the thoracic spine were also elderly individuals. Considering these very distinct characteristics from the current work in relation to the others above, in which attended children and young people with curvatures within the pattern of normality (average of $44.3^{\circ}$ ), believes that there is still a lack of evidence as to the KI of children and adolescents. Thus, it is speculated that new studies, the samples of children and young people present severe increase in the dorsal curvature in which are necessary to test the KI ability in identifying the increased curves, such as the studies with the elderly people.

Nevertheless, an important aspect to be observed is the viability of using the flexicurve, after an initial assessment with the X-rays examination in monitoring the progress of the curvature of the children and the young people, because the results of the angle calculated from KIX (ÂngKIX) and the 
angles obtained with the flexicurve (ÂngKIFint and ÂngKIFT12) were similar (Table 1). This way, the young people would not be exposed to the deleterious effects of radiation and the professional could have a greater control over the evolution of the patient.

In summary, the kyphosis index obtained in the $\mathrm{X}$-rays examination (KIX) and the angle calculated

\section{References}

1. Voutsinas S, MacEwen G. Sagittal profiles of the spine. Clin Orthop Relat Res. 1986; 210: 235-42.

2. Harrison DE, Harrison DD, Cailliet R, Troyanovich SJ, Janik TJ, Holland B. Cobb method or Harrison posterior tangent method: which to choose for lateral cervical radiographic analysis. Spine. 2000; 25: 2072-8.

3. Côt P, Cassidy JD, Yong-Hing K, Sibley J, Loewy J. Apophysial joint degeneration, disc degeneration, and sagittal curve of the cervical spine. Spine. 1997; 22: 85964.

4. Gross C, Gross M, Kuschner S. Error anlaysis of scoliosis curvature measurement. Bulletin of the Hospital for Joint Diseases Orthopaedics Institute. 1983; XX: 171-7.

5. CHEN YL. Vertebral centroid measurement of lumbar lordosis compared with the Cobb technique. Spine. 1999; 24: 1786.

6. Zabjek KF, Leroux MA, Coillard C, Rivard CH, Prince F. Evaluation of segmental postural characteristics during quiet standing in control and idiopathic scoliosis patients.Clin Biomech. 2005; 20: 483-90.

7. Turner-Smith AR, Harris JD, Houghton GR, Jefferson RJ. A method for analysis of back shape in scoliosis. Journal of Biomechanics. 1988; 21: 497-509.

8. de Oliveira TS, Candotti CT, La Torre M, Pelinson PPT, Furlanetto TS, Kutchak FM, Loss JF. Validity and reproducibility of the measurements obtained using the flexicurve instrument to evaluate the angles of thoracic and lumbar curvatures of the spine in the sagittal plane. Rehabil Res Pract. 2012; 2012: 186156.

9. Furlanetto TS, Candotti CT, Comerlato T, Loss JF. Validating a postural evaluation method developed using a Digital Image-based Postural Assessment (DIPA) software. Comput Methods Programs Biomed. 2012; 108: 203-12.

10. Santos Minossi CE, Candotti CT, Bacchi C, Noll M, Casal MZ. Avaliação da coluna lombar e torácica nas situações com salto alto e com os pés descalços com o instrumento arcômetro. Fisioter Pesq. 2012; 19 (3): 196-203. from KIX (ÂngKIX) presented as a method, was accurate and valid to be used in the evaluation of thoracic kyphosis in children and young people. However, the linear and angular values obtained with the flexicurve (KIFint, KIFT12, ÂngKIFint, ÂngKIFT12) showed no correlation with the gold standard, it is not indicated to evaluate thoracic kyphosis in children and young people.

11. Hinman MR. Comparison of thoracic kyphosis and postural stiffness in younger and older women. Spine J. 2004; 4 (4): 413-7.

12. Harrison DE, Cailliet R, Harrison DD, Janik TJ, Holland B. Reliability of centroid, Cobb, and Harrison posterior tangent methods: which to choose for analysis of thoracic kyphosis. Spine. 2001; 26: e227-e234

13. Greendale GA, Nili NS, Huang MH, Seeger L, Karlamangla AS. The reliability and validity of three nonradiological measures of thoracic kyphosis and their relations to the standing radiological Cobb angle. Osteoporosis international. 2011; 22: 1897-1905.

14. Callegari-Jacques SM. Bioestatística: princípios e aplicações. Porto Alegre: Artmed; 2007.

15. Harrison DE, Haas JW, Cailliet R, Harrison DD, Holland $\mathrm{B}$, Janik TJ. Concurrent validity of flexicurve instrument measurements: sagittal skin contour of the cervical spine compared with lateral cervical radiographic measurements. J Manip Physiol Ther. 2005; 28: 597-603.

16. McFarland C, Wang-Price S, Richard S. Clinical measurements of cervical lordosis using flexirule and inclinometer methods in individuals with and without cervical spine dysfunction: A reliability and validity study. J Back Musculoskelet Rehabil. 2015; 28 (2): 295-302.

17. Caine MP, Mcconnell AKE, Taylor D. Assessment of spinal curvature: an evaluation of the flexicurve and associated means of analysis. Int J Rehabil Res. 1996; 19: 271-8.

18. Carman DL, Browne RH, Birch JG. Measurement of scoliosis and kyphosis radiographs: intraobserver and interobserver variation. J Bone Joint Surg [Am]. 1990; 72: 328-33.

19. Teixeira FA, Carvalho GA. Confiabilidade e validade das medidas de cifose torácica através do método flexicurva. Rev Bras Fisioter. 2007; 11: 199-204.

20. Lojudice DC, Lavorato CS, Pereira GP, Cardoso JR. Avaliação da curvatura dorsal em mulheres idosas pelo índice da cifose torácica. Fisioter Pesq. 2006; 13: 32-7.
Received on April 5, 2016

Final version presented on February 5, 2017

Approved on February 14, 2017 